\title{
Destressing MOSFETs by Series Connection in Low Gain Buck Converters
}

This paper was downloaded from TechRxiv (https://www.techrxiv.org).

\section{LICENSE}

CC BY 4.0

SUBMISSION DATE / POSTED DATE

$08-12-2021 / 12-12-2021$

CITATION

Issa, Walid; Ortiz-Gonzalez, Jose; Hu, Yihua (2021): Destressing MOSFETs by Series Connection in Low Gain Buck Converters. TechRxiv. Preprint. https://doi.org/10.36227/techrxiv.17139452.v1

DOI

10.36227/techrxiv.17139452.v1 


\title{
Destressing MOSFETs by Series Connection in Low Gain Buck Converters Walid Issa $^{1^{*}}$, Jose Ortiz-Gonzalez ${ }^{2}$, Yihua $\mathrm{Hu}^{3}$ \\ ${ }^{1}$ Sheffield Halam University, Sheffield, UK \\ ${ }^{2}$ University of Warwick, Coventry, $U K$ \\ ${ }^{3}$ University of York, York, UK \\ *walid.issa@shu.ac.uk
}

Keywords: SERIES, SIC MOSFET, GATE DRIVING, BUCK CONVERTER

\begin{abstract}

\section{Introduction}

Wide-bandgap semiconductors devices enabled more improvements in the power electronics fields applications [1]. However, Silicon Carbide (SiC) MOSFETs provides high switching speed due to low gate capacitance, high breakdown voltage and low thermal resistance, which makes it strong candidate for medium and high voltage applications. However, Commercially available SiC MOSFETs are limited to $1700 \mathrm{~V}$ breakdown voltage devices. Higher voltage MOSFETs are still in the development or costly [2].
\end{abstract}

Low-gain buck converters will enable low voltage loads to access high voltage DC sources by a single stage converter at very low duty cycles. SiC MOSFETs are still limited to $1.7 \mathrm{kV}$ commercially and by seriesing them with adequate gate driving strategy, high voltages can be switched. This paper proposes a driving circuit for series SiC MOSFETs to block higher voltages. The driving circuit provides negative off-state voltage and turn on/off transitions in less than 100ns. The low-gain buck converter performance is assessed when using a single IGBT switch and series SiC MOSFETs. A simulation is implemented and shows the superiority of the proposed driven series SiC MOSFETs with distributed voltage and thermal stresses.

According to the work in [3], series-connected SiC MOSFETs features lower ON-resistance, higher current density, and stronger terrestrial cosmic radiation immunity than using a single high-voltage device. Therefore, the series connection of multiple SiC MOSFETs is reasonable to be investigated.

Medium Voltage DC Network (MVDC) have a huge potential in the coming future to be adopted and implemented in DC grids, for example, to couple power from offshore wind turbines to the national grid via power electronics inverters. The access of these voltages by low voltage loads still not enabled.

Fully separated drivers for $\mathrm{SiC}$ device equipped with isolated power supply is presented in [4] to drive series connected devices. This provides driving flexibility but challenge the power design and compactness of the design. A floating selfdriving circuit is developed in [5] and [6] unitizing the DC capacitors to support switching the high side of two series switches.

Another way of driving series MOSFETs is by using the capacitive coupling [7], where the charge of a capacitor is used as a buffer to generate a driving signal to the next MOSFETs. This design facilitates using a single driving circuit.

For higher gate immunity, it is recommended to switch off the $\mathrm{SiC}$ MOSFET by a negative voltage., i.e. $-5 \mathrm{~V}$. This provides more margin against false turn-on when the temperature of the MOSFET increases, and the gate voltage threshold decreases [8].

This paper will introduce how the series SiC MOSFET enables the access of very high voltages to obtain low voltages. The proof of concept will be implemented using a buck converter where the switch is exposed to high voltage stress. The paper shows a design of a series SiC MOSFET driving circuit. A three series SiC MOSFETs and a single IGBT switch are used in the buck converter design and compared by investigating the stresses on the series MOSFETs and IGBT and the distributed losses while stepping down $3 \mathrm{kV}$ to $48 \mathrm{~V}$.

\section{The proposed Series SiC Driver}

Fig. 1 shows the structure of the driving circuit for series MOSFETs. The circuit here servers for three MOSFETs with a single gate driver. The load is assumed to be inductive as the double pulse testing will be implemented for switching assessment.

Fig. 2 shows the turn on and turn off transition stages as well as the steady off-state of the proposed circuit.

\subsection{Turn-on operation}

Before turning on the MOSFETs, it is assumed that all MOSFETs were in a complete off-state. Therefore, all capacitors have initial charges. Fig. 2a illustrates how the turn on starts by providing the first MOSFET M1 with a driving signal $V_{G}$. This will charge the gate capacitance leading the 
MOSFET M1 to decrease the voltage drop between its drain and source as shown as Loop1. At the same time the driving signal from $V_{G}$ will be coupled through the capacitor $C_{M 1}$ to the gate of the second MOSFET M2 as denoted by Loop2. The drain D1 voltage is dropping while the signal delivered to the gate of M2 becoming sufficient to turn on M2. When MOSFET M2 becomes on, Loop3 shows the charge of $C_{M 2}$ is delivered to the gate of MOSFET M3 through the on-state resistance of M2. At ON steady state, all MOSFETs ae represented by their on-state resistances and the current flows from D3 to S1.

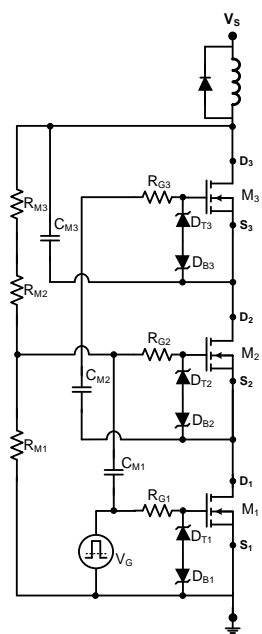

Fig. 1. Driving Series SiC MOSFETs
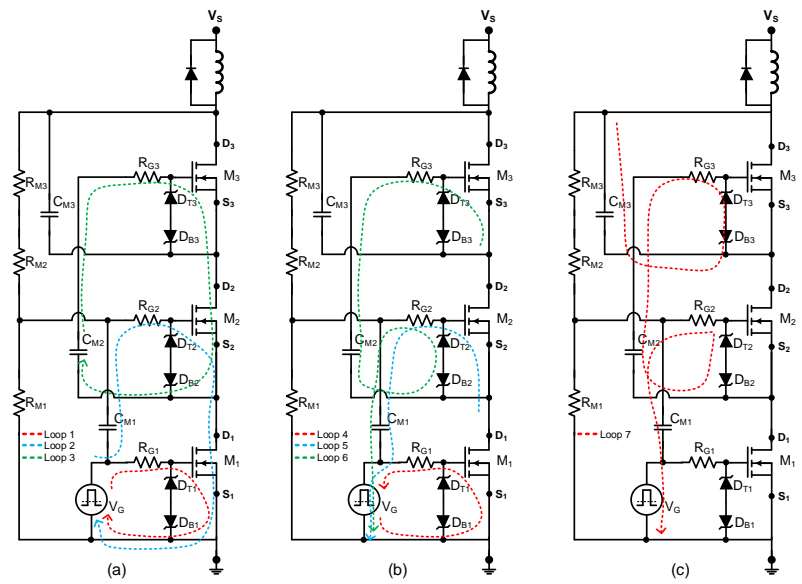

Fig. 2. (a) Turn-on Switching transition, (b) Turn-off Switching transition, (c) Steady off-state

\subsection{Turn-off operation}

Fig. $2 b$ depicts the discharging paths of all gate capacitances. The operation is initiated by an off-voltage signal provided by the gate driver $V_{G}$. As shown by Loop4, the gate of MOSFET M1 will discharge and turning it off. The source node voltage at S2 of MOSFET M2 will rise leading to pushing the charge of the gate capacitance of M2 back to $V_{G}$ through the capacitive coupling $C_{M 1}$ as shown by Loop5. Similar behaviour to turn off M3 through the cooling capacitors $C_{M 1}$ and $C_{M 2}$.

The steady off-state is shown in Fig. 2c. The voltage across M1 is dominated by the ratio between $R_{M 1}:\left(R_{M 2}+R_{M 3}\right)$.
Loop7 shows that the turn-off voltage for MOSFETs M2 and M3 are set by the voltage drop created by the Zener diodes $D_{T}$ and $D_{B}$. The leakage currents of $C_{M 1}, C_{M 2}$ and $C_{M 3}$ are used to bias these Zener diodes and maintain a negative voltage between the gate and source while in off-state.

$$
\begin{aligned}
& I_{\text {leakage }}>I_{z(\min )} \\
& =\frac{V_{s}-(n-1) \times\left(V_{D_{T}}+V_{D_{B}}\right)-V_{\text {drive-off }}}{(n-1) \times R_{G}}
\end{aligned}
$$

where $n$ is the number of MOSFETs and here is $3, V_{\text {drive-off }}$ is the off-state voltage of the gate driver, $V_{D_{T}}, V_{D_{B}}$ are the voltage drop across the Zener diodes in operation and $R_{G}$ is the gate resistance.

The steady state for all MOSFETs are as follows, assuming that the voltage across the resistor $R_{G}$ is neglected,

$$
V_{o f f-M 1}=V_{S} \times \frac{R_{M 1}}{R_{M 1}+R_{M 2}+R_{M 3}}-\left(V_{D_{T}}+V_{D_{B}}\right)
$$

The voltage across $C_{M 2}$ and $C_{M 3}$ are determined by

$$
\begin{aligned}
V_{\left(R_{M 2}+R_{M 3}\right)}=V_{s} \times & \frac{R_{M 2}+R_{M 3}}{R_{M 1}+R_{M 2}+R_{M 3}} \\
& =V_{C_{M 3}}+\left(V_{D_{T 3}}+V_{D_{B 3}}\right)+V_{C_{M 2}} \\
& +\left(V_{D_{T 3}}+V_{D_{B 3}}\right)
\end{aligned}
$$

Here it is assumed that $V_{C_{M 2}}=V_{C_{M 3}}$ due to similarity, Therefore,

$$
\begin{gathered}
V_{o f f-M 2}=V_{C_{M 2}}+\left(V_{D_{T}}+V_{D_{B}}\right) \\
V_{o f f-M 3}=V_{C_{M 3}}
\end{gathered}
$$

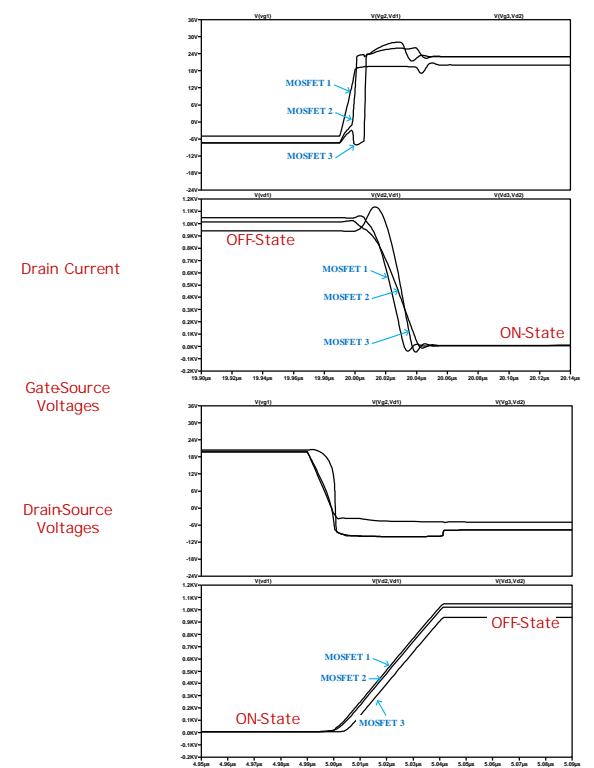

Fig. 3. Double pulse testing and its zoomed view for the turnon and turn-off transitions.

Table 1 Parameter for the DPT simulation

\begin{tabular}{|l|l|l|l|}
\hline Component & Value & Component & Value \\
\hline$V_{S}$ & $3000 \mathrm{~V}$ & $D_{B}$ & $6.8 \mathrm{~V}$ \\
\hline$L$ & $1000 \mu \mathrm{H}$ & $D_{T}$ & $22 \mathrm{~V}$ \\
\hline$R_{M}$ & $100 \mathrm{k} \Omega$ & $R_{G}$ & $6 \Omega$ \\
\hline$C_{M}$ & $200 \mathrm{pF}$ & $V_{\text {drive-on }}$ & $20 \mathrm{~V}$ \\
\hline MOSFETs & $\mathrm{C} 2 \mathrm{M} 1000170 \mathrm{D}$ & $V_{\text {drive-off }}$ & $-5 \mathrm{~V}$ \\
\hline
\end{tabular}




\section{Double Pulse Testing Simulation}

LTspice was used to simulate switching three MOSFETs in series as in Fig. 1 with a clamped inductor load. The modelled SiC MOSFET is C2M1000170D. Wolfspeed provides the spice model. the simulation values are shown in Table 1. The DPT waveforms are shown in Fig. 3 including the gatesource voltages and the drain-source voltages. The results depict a semi-balanced voltage sharing at off- steady state due to the existence of Zener voltages. In addition, it shows consistent dynamics of voltage and currents among all MOSFETs.

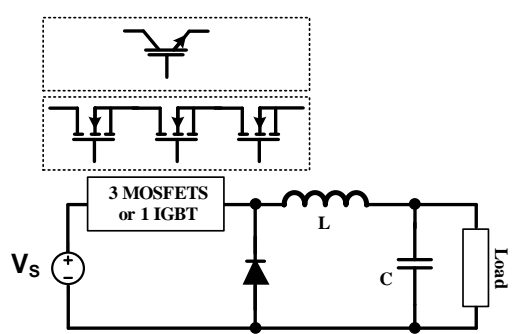

Fig. 4. Low gain buck converter

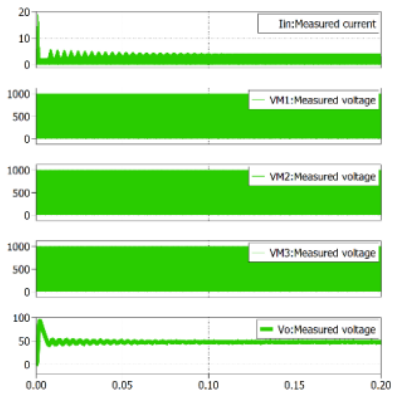

(a)
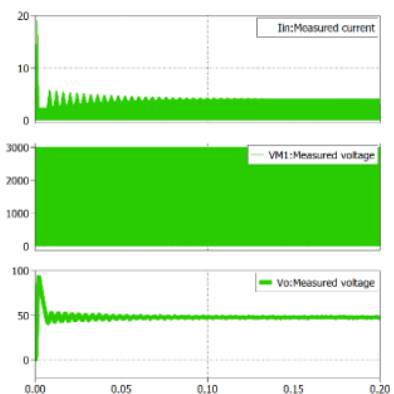

(c)

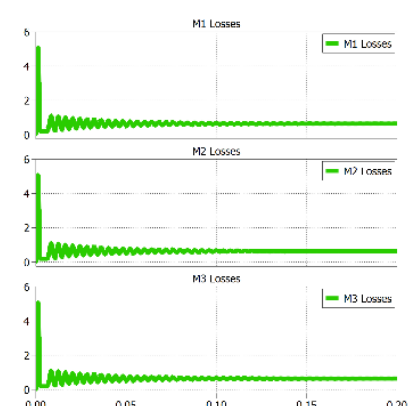

(b)

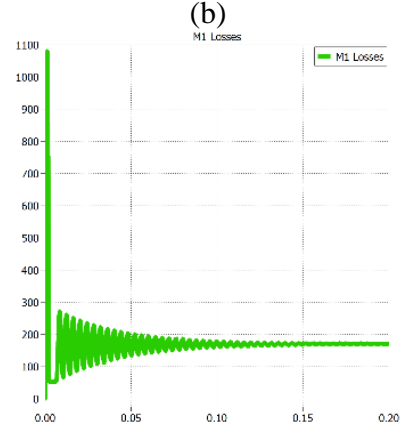

(d)
Fig. 5. (a) Output voltage and current and voltage stresses on the three MOSFETs (b) Total losses of each MOSFET (c) Output voltage and current and voltage stresses on the IGBT (b) Total losses of the IGBT

\section{Simulation of Low Gain Buck Converter}

The design of buck converter components is well-established in the literature and here the focus is on the performance of the power switch in the buck converter which is supplied by a high voltage as $3000 \mathrm{~V}$ as shown in Fig. 4. The desired output voltage is $48 \mathrm{~V}$ and load current is $3 \mathrm{~A}$. Therefore, the calculated steady state duty cycle is $1.6 \%$. PLECS has been used to conduct the simulation where each power switch is equipped with similar heatsink. The inductor is $2 m \mathrm{H}$ and capacitor is $200 \mu \mathrm{F}$. The diode is capable to handle the voltage stress. The PLECS models of the used power switches are provided by the manufacturers. The simulated results are shown in Fig. 5 for the output voltage, current/voltage stresses in the power switches and total losses including the switching and conduction loss. The switching frequency is selected as $10 \mathrm{kHz}$. A three series SiC MOSFETS with braking voltage of $1.2 \mathrm{kV}, \mathrm{C} 2 \mathrm{M} 1000170 \mathrm{D}$, are used where their voltage stresses are shown in Fig. 5a, as $1 \mathrm{kV}$ per MOSFET, and losses in Fig. $5 \mathrm{~b}$ as $0.64 \mathrm{~W}$ per MOSFET. The output voltage is stable at $48 \mathrm{~V}$. Similarly, the three MOSFETS are replaced with a $3.3 \mathrm{kV}$ IGBT, FZ1200R33HE3, and the simulation results are shown in Fig. 5c where the voltage stress is $3 \mathrm{kV}$ and losses are calculated as $168.5 \mathrm{~W}$ in Fig. 5d. It is surprisingly shown that the series MOSFETs outperform the IGBT switch.

\section{Conclusion}

In this paper, a low gain buck converter is used to assess the performance of series SiC MOSFET against a high breaking voltage IGBT to step down $3000 \mathrm{~V}$ to $48 \mathrm{~V}$. Each MOSFET is able to block $1200 \mathrm{~V}$. A driving circuit is proposed with a single gate driver to be able to switch on/off the three MOSFETs. The double pulse testing validated the operation of the driving circuit. The buck converter shown high performance when it used the three MOSFETs compared with a single IGBT. In addition, the electrical and thermal stresses have been distributed leading to longer lifetime and less colling requirement.

\section{References}

[1] J. Millan, P. Godignon, X. Perpina, A. Perez-Tomas, and J. Rebollo, "A survey of wide bandgap power semiconductor devices," IEEE Trans. Power Electron., vol. 29, no. 5, pp. 2155-2163, May 2014, doi: 10.1109/TPEL.2013.2268900.

K. Mainali et al., "A Transformerless Intelligent Power Substation: A three-phase SST enabled by a $15-\mathrm{kV}$ SiC IGBT," IEEE Power Electron. Mag., vol. 2, no. 3, pp. 31-43, Sep. 2015, doi: 10.1109/MPEL.2015.2449271.

[3] A. Bolotnikov et al., "Overview of $1.2 \mathrm{kV}-2.2 \mathrm{kV} \mathrm{SiC} \mathrm{MOSFETs}$ targeted for industrial power conversion applications," Conf. Proc. - IEEE Appl. Power Electron. Conf. Expo. - APEC, vol. 2015-May, no. May, pp. 2445-2452, May 2015, doi: 10.1109/APEC.2015.7104691.

S. Ji, T. Lu, Z. Zhao, H. Yu, and L. Yuan, "Series-Connected HVIGBTs Using Active Voltage Balancing Control with Status Feedback Circuit," IEEE Trans. Power Electron., vol. 30, no. 8, pp. 4165-4174, 2015, doi: 10.1109/TPEL.2014.2360189.

P. Grbović, "High-voltage auxiliary power supply using seriesconnected MOSFETs and floating self-driving technique," IEEE Trans. Ind. Electron., vol. 56, no. 5, pp. 1446-1455, 2009, doi: 10.1109/TIE.2009.2012418.

[6] P. J. Grbović, "Loss-free balancing circuit for series connection of electrolytic capacitors using an auxiliary switch-mode power supply," IEEE Trans. Power Electron., vol. 24, no. 1, pp. 221-231, 2009, doi: 10.1109/TPEL.2008.2005579.

[7] H. L. Hess and R. J. Baker, "Transformerless capacitive coupling of gate signals for series operation of power MOS devices," IEEE Trans. Power Electron., vol. 15, no. 5, pp. 923-930, Sep. 2000, doi: $10.1109 / 63.867682$. 
[8] Y. Ren et al., "A Compact Gate Control and Voltage-Balancing Circuit for Series-Connected SiC MOSFETs and Its Application in a DC Breaker," IEEE Trans. Ind. Electron., vol. 64, no. 10, pp. 8299-8309, Oct. 2017, doi: 10.1109/TIE.2017.2711579. 